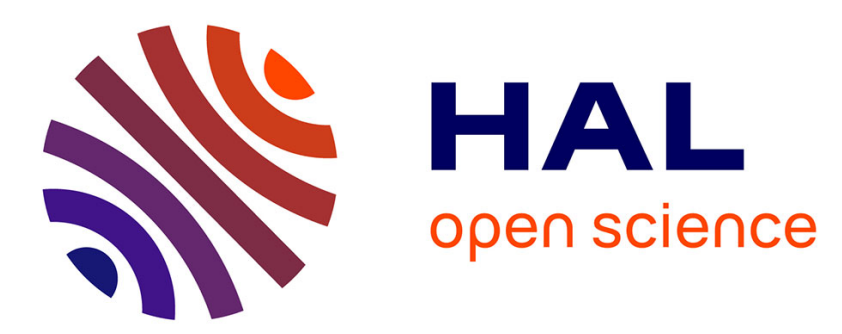

\title{
Étude comparative de différents correcteurs pour la commande optimale avec défauts d'une machine pentaphasée
}

Fatiha Mekri, Jean-Frederic Charpentier, Xavier Kestelyn, Eric Semail

\section{To cite this version:}

Fatiha Mekri, Jean-Frederic Charpentier, Xavier Kestelyn, Eric Semail. Étude comparative de différents correcteurs pour la commande optimale avec défauts d'une machine pentaphasée. European Journal of Electrical Engineering, 2012, 15 (4), pp.377-400. 10.3166/ejee.15.377-400 . hal-00804468

\section{HAL Id: hal-00804468 \\ https://hal.science/hal-00804468}

Submitted on 25 Mar 2013

HAL is a multi-disciplinary open access archive for the deposit and dissemination of scientific research documents, whether they are published or not. The documents may come from teaching and research institutions in France or abroad, or from public or private research centers.
L'archive ouverte pluridisciplinaire HAL, est destinée au dépôt et à la diffusion de documents scientifiques de niveau recherche, publiés ou non, émanant des établissements d'enseignement et de recherche français ou étrangers, des laboratoires publics ou privés. 


\title{
Etude comparative de différents correcteurs pour la commande optimale avec défauts d'une machine pentaphasée
}

\author{
F. Mekri* - J-F. Charpentier*_- X. Kestelyn**, E. Semail** \\ *BCRM Ecole Navale et des Groupes du Poulmic CC 600-29240 BREST, France \\ Jean-frederic.charpentier@ecole-navale.fr \\ ** L2EP, ENSAM. 8, boulevard Louis XIV 59046 Lille cedex
}

\begin{abstract}
RÉSUMÉ. Cet article est consacré à l'étude d'une méthode de commande d'une machine synchrone à aimant permanent à cinq phases associée à un onduleur de tension à MLI. On $s$ 'intéresse au cas de la commande en mode normal et en mode dégradé. Les défauts étudiés sont liés à la déconnexion d'une phase ou de plusieurs phases. Dans ce cas, la méthode étudiée est basée sur une approche vectorielle permettant la détermination en ligne des courants de référence optimaux adaptés au défaut. L'étude se propose de comparer l'utilisation de régulateurs PID avec des régulateurs non linéaire de type hystérésis à bande fixe et à bande adaptative. Cette comparaison est effectuée en mode normal et en mode de défaut. Les résultats de simulation effectués sur MATLAB / Simulink sont présentés et discutés afin de vérifier les performances de la stratégie et des régulateurs étudiés. Ils montrent en particulier que si la régulation de type PID est performante en mode normal, elle ne permet pas d'assurer un suivi correct des références en mode de défaut car celles ci présentent des variations dynamiques importantes. Dans ce dernier cas d'étude l'utilisation de commandes non linéaires comme la commande par hystérésis est nécessaire et permet de garder le même schéma de commande en mode normal et dégradé. L'utilisation d'une commande par hystérésis à bande adaptative est alors judicieuse car elle permet de bonnes performances de suivi et une maitrise de la fréquence de commutation de l'onduleur.

ABSTRACT. The use of multiphase PM machines associated with VSI drives appears to be a very efficient solution. Presented work focus on the use of such a system in open circuited phase fault conditions. With this kind of system it is possible to determine optimal currents references which maximize the torque density of the system when one or two phases are open circuited. Classical linear controllers (as PID for example) cannot provide a correct tracking of these optimal reference currents because they have a highly dynamical behavior. We propose in this paper to combine this optimal reference current generation with hysteresis control. This kind of solution allows a good tracking of these unconventional reference currents with a fixed switching frequency for the VSI. In this case of adaptive hysteresis band control appears to be particularly efficient.

MoTS-CLÉS: multi machines, mode en défaut, contrôle non linaire, en ligne stratégie, détermination des courants de références,

KEYWORDS: multi phase machine, fault tolerant, nonlinear control, on line strategy, current reference.
\end{abstract}

Revue. Volume $\mathrm{X}-\mathrm{n}^{\circ} \mathrm{x} /$ année, pages 1 à $\mathrm{X}$ 


\section{Introduction}

L'utilisation des machines synchrones à aimants permanents (MSAP) à grand nombre de phases alimentées par des onduleurs de tension à MLI est particulièrement adaptée à des cahiers des charges exigeants en termes de sécurité de fonctionnement et/ou de discrétion acoustique et/ou de grande puissance. En effet, la multiplication du nombre de phases permet d'une part le fractionnement de puissance et donc la diminution des contraintes sur les composants mais aussi d'autre part la réduction des ondulations de couple et le fonctionnement en mode dégradé (avec une ou plusieurs phases en défaut). De ce fait, ces technologies sont particulièrement adaptées à des contextes comme la propulsion navale, la traction ferroviaire ou l'automobile. Les avancées dans le domaine de l'électronique de puissance et de commande permettent d'assurer des commandes robustes de ces machines quel que soit leur mode fonctionnement. Différents travaux ont été réalisés sur les machines polyphasées alimentées par onduleur de tension (Parsa et al. 2005)(Dwari et al. 1998)- (Levi et al. 2004)-(Locment et al. 2008). Certains de ces travaux se basent sur la décomposition de la machine polyphasée en machines fictives équivalentes et découplées magnétiquement (Locment et al. 2008). En mode dégradé, différentes méthodes ont été présentées (Dwari et al. 1998)- (Locment et al. 2008) -(Martin et al. 2007) afin de supprimer les ondulations de couple générées par la déconnexion d'une ou plusieurs phases d'une MSAP polyphasée. Ces méthodes sont basées sur la modification de la forme d'onde du courant d'une ou plusieurs phases actives de la machine. Pour une structure classique triphasée composée d'un onduleur à q bras, certains auteurs proposent d'ajouter un bras supplémentaire pour contrôler le courant homopolaire (Bolognani et al. 2000). Pour une structure d'alimentation ou chaque phase est associée à un onduleur monophasé, certains auteurs proposent également de modifier l'amplitude des courants dans les phases actives (Martin et al. 2000)- (Martin et al. 2007).

Dans ce travail, après avoir présenté la théorie de modélisation multimachines des machines polyphasées (Semail et al. 2005), nous nous intéresserons à son application à la détermination des formes optimales des références de courants en mode normal et dégradé. Ces références optimales permettent alors de minimiser les ondulations de couple et les pertes Joule. Nous nous intéresserons en particulier à l'application de cette stratégie au cas de la régulation des courants d'une MSAP à 5 phases alimentée par un onduleur de tension à 5 bras (figure 1). Des résultats de simulation seront présentés pour cette machine, en mode normal et en mode dégradé. Le but de la commande est alors de maintenir un couple constant à pertes Joule minimales tout en permettant une gestion en ligne des défauts. Ce travail qui fait suite aux travaux menés dans (Martin et al. 2007) et (Kestelyn et al. 2009), consiste à comparer différents types de stratégie de contrôle pouvant être adaptés à ce mode de commande. Il présente en particulier l'étude de stratégies de contrôle basées sur un contrôle par hystérésis à bande adaptative qui s'avère particulièrement adapté. Nous examinerons ainsi les performances de différents types de correcteurs et en 
particuliers des correcteurs de type PID et des correcteurs par hystérésis à bande fixe et à bande auto-adaptative.

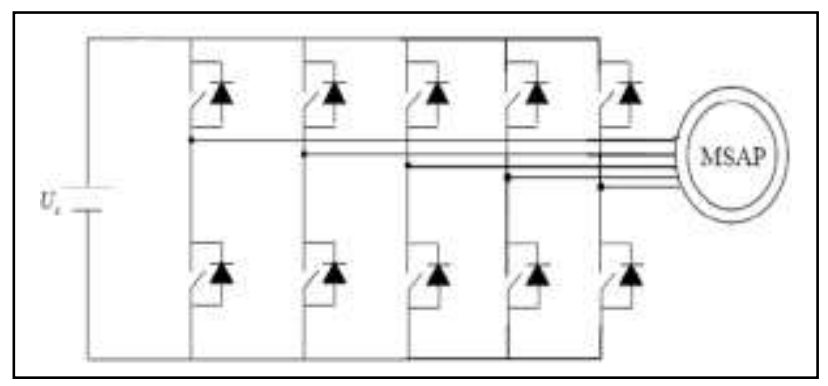

Figure 1. Machine à 5 phases couplée en étoile et alimentée par un onduleur de tension à 5 bras.

\section{Formalisme Multimachine}

Les équations électriques d'une machine synchrone pentaphasée dans la base naturelle (base associée aux 5 phases) sont données pour chaque phase (entre phase et neutre) par l'expression suivante :

$$
v_{s k}=R_{s} i_{k}+\frac{d \phi_{s k}}{d t}+e_{k}
$$

Où $\phi_{s k}$ est le flux dans la phase $k$ créé par les courants statoriques, $e_{k}$ est la Fem induite dans la phase $k$ par le rotor et $V_{s k}$ est la tension d'alimentation aux bornes de la phase $k$. Il est à noter que le système [1] est alors multidimensionnel et fortement couplé ce qui fait qu'il est difficile d'établir des critères de correction et de calculer des correcteurs de manière pertinente. Pour palier à ce problème, on se base sur le caractère symétrique et circulant de la matrice inductance. Cette matrice est en effet diagonalisable dans une base orthonormée. Il est alors possible d'écrire les équations [1] dans cette nouvelle base (en passant de la base naturelle à cette nouvelle base par une transformation dite de Concordia Généralisée) (Kestelyn, 2003)- (Parsa et al. 2007)- (Semail et al. 2004)- (Kestelyn et al. 2011).. On obtient alors les équations caractéristiques de 2 machines diphasées et d'une machine monophasée fictives que l'on peut appeler principale (plan $\alpha_{p}, \beta_{p}$ ), secondaire (plan $\alpha_{s}, \beta_{s}$ ) et homopolaire (droite $z$ ). Ces machines fictives sont caractérisées par des forces électromotrices et des inductances de valeurs différentes: 


$$
\begin{aligned}
& \vec{v}_{z}=R_{s} \vec{i}_{z}+\Lambda_{z} \frac{d \vec{i}_{z}}{d t}+\vec{e}_{z} \\
& \vec{v}_{\alpha \beta-p}=R_{s} \vec{i}_{\alpha \beta-p}+\Lambda_{p} \frac{d \vec{i}_{\alpha \beta-p}}{d t}+\vec{e}_{\alpha \beta-p} \\
& \vec{v}_{\alpha \beta}=R_{s} \vec{i}_{\alpha \beta-s}+\Lambda_{s} \frac{d \vec{i}_{\alpha \beta-s}}{d t}+\vec{e}_{\alpha \beta-s}
\end{aligned}
$$

Toutes les machines fictives (ou les 2 plans et l'axe homopolaire) sont alors découplés magnétiquement et donc on peut définir pour chacun un schéma électrique équivalent indépendant. Chacun de ces systèmes est alimenté par un vecteur de tension $\vec{v}_{x}$ dit soit principal $\left(\vec{v}_{p}\right)$ ou secondaire $\left(\vec{v}_{s}\right)$ ou homopolaire $\left(\vec{v}_{h}\right)$, et comprend une résistance $\left(R_{s}\right)$, une inductance cyclique $\left(\Lambda_{x}\right)$ et un vecteur FEM (principal, secondaire, ou homopolaire). Le schéma électrique équivalent à chacun de ces systèmes est donné par la figure 2, avec $x$ pouvant représenter la machine principale, secondaire ou homopolaire $(x=p, s, h)$. Il est à noter qu'à cette décomposition en sous espaces liée aux machines fictives correspond également à une décomposition harmonique des signaux. En effet, les signaux (forces électromotrices, tensions, courants) se projettent par familles d'harmoniques dans ces sous espaces. Les harmoniques 1,9 et 11 se projettent dans le plan principal, les harmoniques 3, 7 et 13 dans le plan secondaire et les harmoniques multiples de 5 sur la droite homopolaire.

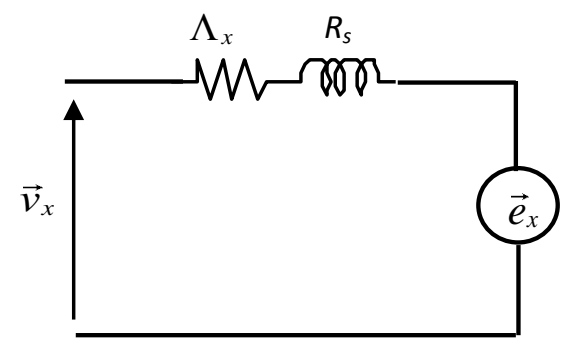

Figure 2. Schéma équivalent d'une phase de la machine

\section{- Expression de la FEM :}

Le vecteur de la FEM dans la base naturelle (5 phases) d'une machine synchrone pentaphasée peut s'écrire sous forme:

$$
\begin{aligned}
\vec{e} & =e_{1} \vec{x}_{1}+e_{2} \vec{x}_{2}+e_{3} \vec{x}_{3}+e_{4} \vec{x}_{4}+e_{5} \vec{x}_{5} \\
\text { Avec }: e_{k} & =\sum_{h=1}^{\infty} E^{k} \sin \left(h\left(p \theta-(k-1) \frac{2 \pi}{5}\right)\right)
\end{aligned}
$$


$E^{h}=k_{f e m}^{h} \Omega$ est la valeur crête de l'harmonique de FEM de rang h.

En projetant le vecteur de la FEM sur les différents sous espaces $[z, \alpha, \beta]$, on obtient les équations du système [4] :

$$
\left.\begin{array}{l}
\vec{e}_{z}=\sqrt{5} \sum_{h} E^{h} \sin (h p \theta) \vec{x}_{z}, \mathrm{~h}=5,15, \ldots \\
\vec{e}_{p}=\sqrt{\frac{5}{2}} \sum_{\mathrm{h}} E^{h}\left(\sin (h p \theta) \vec{x}_{p \alpha}-\cos (h p \theta) \vec{x}_{p \beta}\right), \mathrm{h}=1,9, \ldots \\
\vec{e}_{s}=\sqrt{\frac{5}{2}} \sum_{h} E^{h}\left(\sin (h p \theta) \vec{x}_{s \alpha}+\cos (h p \theta) \vec{x}_{s \beta}\right), \mathrm{h}=3,7, . . \\
\vec{e}=\vec{e}_{p}+\vec{e}_{s}+\vec{e}_{z}
\end{array}\right\}
$$

Les $\vec{e}_{p}, \vec{e}_{s}, \vec{e}_{h}$ correspondent à des vecteurs, obtenus par projection du vecteur force électromotrice dans les espaces $\left(\alpha_{p}, \beta_{p}\right),\left(\alpha_{s}, \beta_{s}\right)$ et $z$ qui sont liés à la transformation de Concordia généralisée. A titre d'exemple nous considérerons une machine 5 phases à aimant permanent de faible puissance. La figure 3 présente la forme d'onde de la FEM mesurée à vide aux bornes de l'une des phases de cette machine à $1250 \mathrm{tr} / \mathrm{mn}$. En se basant sur ce relevé expérimental, on en déduit le contenu harmonique résumé dans le tableau 1 , ce dernier montre que l'harmonique 3 représente pour cette machine $23 \%$ de l'amplitude du fondamental et que l'amplitude de l'harmonique 7 représente moins de $1 \%$ de l'amplitude du fondamental.

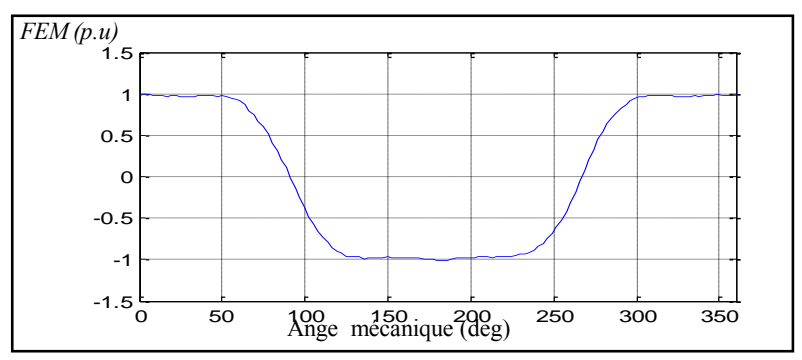

Figure 3. Relevé expérimental d'une FEM. 


\begin{tabular}{|l|l|l|l|l|}
\hline Harmonique & 1 & 3 & 5 & 7 \\
\hline $\begin{array}{l}\text { Taux relatif } \\
\text { en }(\%)\end{array}$ & 100 & 23 & 7.31 & 0.82 \\
\hline
\end{tabular}

Tableau 1. Contenu harmoniques des FEMs

\section{- Expression du couple électromagnétique :}

Les sous espaces associés aux machines fictives étant orthogonaux, la puissance électromagnétique est le produit scalaire des vecteurs FEM et courant et le couple peut donc être vu comme la somme des couples des machines principale, secondaire et homopolaire.

$$
C_{e m}=\frac{\vec{e} \cdot \vec{i}}{\Omega}=\frac{\vec{e}_{z} \vec{i}_{z}+\vec{e}_{p} \cdot \vec{i}_{p}+\vec{e}_{s} \cdot \vec{i}_{s}}{\Omega}
$$

Il est à noter que si un couplage étoile est établi, ce qui est le cas pour notre étude, la machine homopolaire n'est pas alimentée et ne peux fournir de couple. Cela revient à écrire le couple sous forme :

$$
\begin{gathered}
C_{e m}=\frac{\vec{e}_{p} \cdot \vec{i}_{p}+\vec{e}_{s} \cdot \vec{i}_{s}}{\Omega} \\
C_{e m}=C_{p}+C_{s}
\end{gathered}
$$

D'autre part, l'équation mécanique de la machine est :

$$
C_{e m}=j \frac{d \Omega}{d t}+f \Omega
$$

On constate alors qu'avec une alimentation en courant adaptée les machines principale et secondaire sont capables chacune de contribuer au couple de la machine réelle si les projections de la FEM dans chacun des sous espaces correspondants est non nulle ce qui est le cas de la machine étudiée qui présente une FEM contenant une part significative de $3^{\text {ème }}$ harmonique. 


\section{Stratégie d'alimentation et de commande en mode normal.}

$\mathrm{Au}$ vu de la répartition harmonique dans les sous espaces on peut alors considérer au niveau des premiers harmoniques projetés dans chaque sous espace que, la machine principale possède un nombre de paires de pôles égale à $p$ (nombre de paires de pôles de la machine réelle) et que la machine secondaire a 3 fois ce nombre de paires de pôles. Une solution intéressante en termes de contrôle est d'alimenter la machine principale et secondaire par des courants « sinusoïdaux » qui correspondent chacun au premier harmonique de la famille d'harmoniques des deux sous-espaces associés. Ceci revient à injecter en plus d'un courant sinusoïdal correspondant au fondamental (premier harmonique de la machine principale) un courant harmonique d'ordre 3 qui correspond au premier harmonique de la machine secondaire. Cependant un bon suivi en dynamique est alors difficile à obtenir car en régime permanent les références varient avec une dynamique liée à la vitesse de la machine. Afin de simplifier la conception d'une commande dynamique, il est alors possible d'appliquer la transformation de Park appropriée à chaque machine diphasée (d'angle $\theta$ pour la machine principale et d'angle $-3 \theta$ pour la machine secondaire). Ceci permet de contrôler le courant dans chaque machine (et donc leur contribution au couple) indépendamment en utilisant, en entrée des régulateurs, des références de courant qui sont constantes en régime permanent.

En mode normal, pour optimiser la compacité de la machine, on cherche souvent à minimiser les pertes Joule à couple maximum et constant (Martin et al. 2007). Pour ce faire, il est nécessaire que le courant $\vec{i}$ soit colinéaire à $\vec{e}$, cela revient à imposer des ondes de courant de même formes que la FEM et en phase avec cette FEM (équations [7]). Dans ce cas, le couple électromagnétique est maximum à pertes Joule données (ou pour un couple donné, les pertes Joule sont minimisées). Dans le cas de la stratégie définie précédemment, cela revient à injecter pour la machine 5 phase un courant harmonique de rang 3 dont le rapport avec le fondamental du courant respecte le rapport des harmoniques 3 et 1 de la FEM. Dans le cas de la machine étudiée l'amplitude du courant d'harmonique 3 sera donc maintenue à $23 \%$ de la valeur de celle du courant fondamental.

$$
\begin{aligned}
& \vec{i}=k \frac{\vec{e}}{\Omega} \\
& \text { avec } C_{\max }=\frac{\vec{e}}{\Omega} \vec{i}=\frac{\vec{e}}{\Omega}\left(k \frac{\vec{e}}{\Omega}\right)=k\left\|\frac{\vec{e}}{\Omega}\right\|^{2} \\
& \text { D'où : } \quad k=\frac{C_{\max }}{\left\|\frac{\vec{e}}{\Omega}\right\|^{2}} \Rightarrow \vec{i}_{\text {ref }}=k \frac{\vec{e}}{\Omega}
\end{aligned}
$$




\section{Stratégie de commande en mode dégradé}

Si la stratégie précédente est appliquée telle quelle en mode dégradé, elle se traduit par un couple pulsatoire important de pulsation $2 \omega$ et par une diminution du couple moyen (Martin et al. 2007). Pour y remédier il faut imposer une nouvelle stratégie de calcul des références de courant. Cette méthode consiste à modifier la forme d'onde des références de courants de toutes les phases actives de sorte que le couple pulsatoire soit minimisé. On maintient la colinéarité entre le vecteur courant et un nouveau vecteur force électromotrice modifié que l'on appelle $(e$ '), constitué des phases alimentées à l'exclusion des phases non alimentées afin de minimiser les pertes Joule (Martin et al. 2007)- (Kestelyn et al. 2009). Après détection des phases en défaut, on est alors amené à considérer un nouveau système (dont les variables sont notées $x^{\prime}$ ) au nombre de phases égales au nombre de phases initiales moins le nombre de phases en défauts (Kestelyn et al. 2009). Dans ce cas, le couple est le produit scalaire du vecteur courant et du vecteur FEM modifié $\left(\vec{e}^{\cdot}\right)$. L'expression de la FEM modifiée d'une phase active, par exemple celle de la phase $1, e_{1}$ 'est alors donnée par l'équation suivante:

$$
e_{1}^{\prime}=e_{1}-\frac{1}{q^{\prime}} \sum_{j=1}^{q} h_{j} e_{j}
$$

$q$ ' est le nombre de phases actives, $h_{j}=1$ si la phase $\mathrm{j}$ est active, et $h_{j}=0$ si la phase $\mathrm{j}$ est inactive. La FEM modifiée des phases en défaut est quand à elle fixée à 0 .

Cette transformation permet alors de supprimer la composante homopolaire de la FEM des phases actives.

On peut alors obtenir les nouvelles références de courant de la manière suivante :

$$
k^{\prime}=\frac{C_{\max }}{\left\|\frac{\vec{e}^{\prime}}{\Omega}\right\|^{2}} \Rightarrow \vec{i}^{\prime}=K^{\prime} \frac{\vec{e}^{\prime}}{\Omega}
$$

Cette stratégie reste valable en mode normal et en mode dégradé pour l'obtention d'un couple constant à pertes Joule minimales. Elle permet donc une gestion en temps réel des défauts du type ouverture de phase sans reconfiguration de la structure de commande. La structure générale de génération de ces courants de référence qui est valable en mode normal et dégradé est illustrée par la figure.4. 


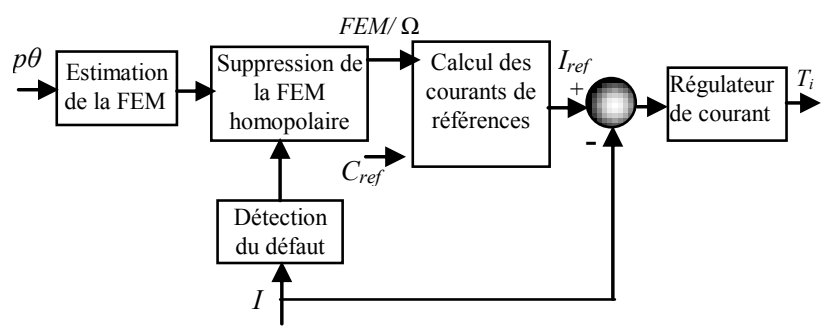

Figure 4. Stratégie de génération des courants de référence de la machine polyphasée

\section{Contrôle des courants d'un MSAP polyphasé}

\subsection{Contrôle linéaire (Régulateur PID).}

Comme expliqué précédemment la commande en mode normal se fait en appliquant la transformation de Park appropriée à chaque machine diphasée donnée par le système [2]. On peut alors considérer qu'en mode normal chacune des machines fictives a des références correspondant à des courants sinusoïdaux en régime permanent. Le schéma bloc de contrôle de la machine principale dans le référentiel de Park, est donné par la figure 5. Les grandeurs électriques dans les axes directs, $\mathrm{d}$, et transverses, $\mathrm{q}$, de la machine principale sont alors obtenues à partir des grandeurs projetées dans le sous espace principal en appliquant la matrice de rotation classique d'angle $\theta(\mathrm{P}(\theta))$. On a la même structure de contrôle pour la machine secondaire suivant l'axe $\mathrm{q}$ ou $\mathrm{d}$ en appliquant la matrice de rotation classique $\mathrm{P}(-3 \theta)$ pour les grandeurs projetés dans le plan de la machine secondaire. On obtient alors le système [9].

$$
\left.\begin{array}{l}
v_{d p}=R_{s} I_{d p}-\omega \Lambda_{p} I_{q p}+E_{d p}+\Lambda_{p} \frac{d I_{d p}}{d t} \\
v_{q p}=R_{s} I_{q p}+\omega \Lambda_{p} I_{d p}+E_{q p}+\Lambda_{p} \frac{d I_{q p}}{d t} \\
v_{d s}=R_{s} I_{d s}-3 \omega \Lambda_{s} I_{q s}+E_{d s}+\Lambda_{s} \frac{d I_{d s}}{d t} \\
v_{q s}=R_{s} I_{q s}+3 \omega \Lambda_{s} I_{d s}+E_{q s}+\Lambda_{s} \frac{d I_{q s}}{d t}
\end{array}\right\}
$$


Dans le système [9], les termes introduits par la rotation due à la transformation de Park et les FEMs sont considérés comme des perturbations qui sont compensées sans difficulté. A part, les perturbations introduites par les ondulations de la FEM de la machine secondaire $\left(E_{d s}, E_{q s}\right)$ qui ont un caractère non sinusoïdal et dépendent de l'angle de transformation, on suppose que ces perturbations ont un effet négligeable vis à vis la boucle de régulation. Il est alors possible de contrôler le couple de chacune des deux machines (principale et secondaire) en utilisant un contrôle par un correcteur «classique » de type PID. Les gains de ce correcteur sont choisis tels que la fréquence de coupure du système en boucle fermé (second ordre) soit $\mathrm{f}_{0}=\omega_{0} / 2 \pi=$ $1000 \mathrm{~Hz}$ et que son amortissement $\xi$ soit égal à 0.7 .

Il est à noter que si en mode normal et en régime permanent, les transformations de Park associées aux deux machines permettent d'effectuer un contrôle avec des courants de référence dont la dynamique n'est pas directement liée à la vitesse de rotation de la machine (figure $6(\mathrm{a}, \mathrm{b})$ ), ce n'est plus le cas lors de l'apparition d'un défaut. En effet [8] conduit alors à obtenir des références de courants dont la dynamique est fonction de la vitesse de rotation de la machine. Il est à noter que cette dynamique est d'autant plus élevée une fois les transformations de Park appliquées. A titre d'exemple la figure $7(a, b)$ présente la forme d'onde des références de courants de la machine étudiée ramené à sa valeur unitaire (en per unit, p.u) lorsqu'une phase est déconnectée dans l'espace réel et dans les espaces de Park associées aux deux machines. Ainsi si nous souhaitons conserver un même schéma de commande en mode normal et dégradé, une régulation de type PID peut vite être limitée aux fonctionnements à basses vitesses en termes de suivi des références, lorsqu'un défaut apparait. C'est pourquoi nous proposons dans cet article de comparer cette régulation avec des modes de régulation plus adaptés à ce caractère dynamique en mode de défaut. Dans ce cadre nous comparerons l'usage de régulateurs de type PID avec celui de régulateurs à hystérésis utilisant une bande fixe et une bande auto adaptative. Cette comparaison permettra de définir selon le cahier des charges à partir de quelle vitesse le changement de correcteur peut devenir intéressant.

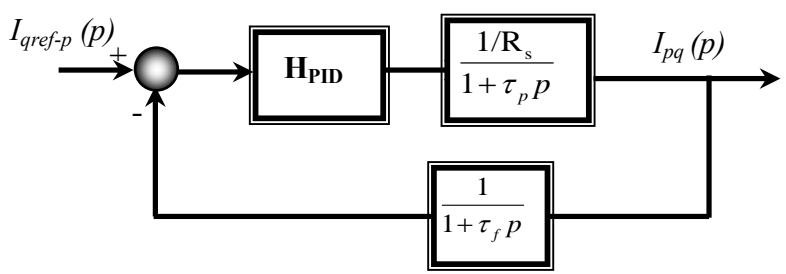

Figure 5. Schéma fonctionnel de la régulation du courant (Iq-p) 


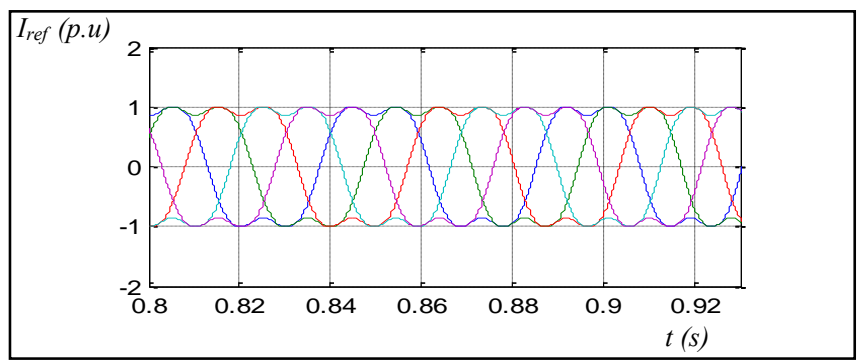

Figure 6 (a). Référence de courant (en p.u.) en mode normal base naturelle

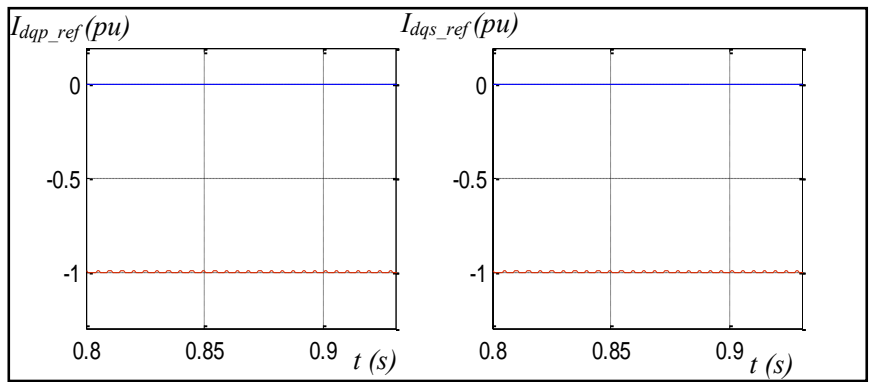

Figure 6(b). Référence de courant (en p.u.) en mode normal dans le référentiel de Park

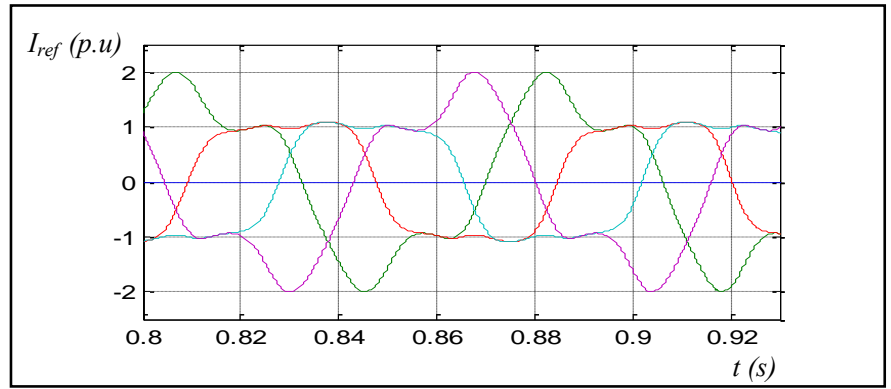

Figure 7 (a). Référence de courant (en p.u.) avec une phase ouverte dans la base naturelle 


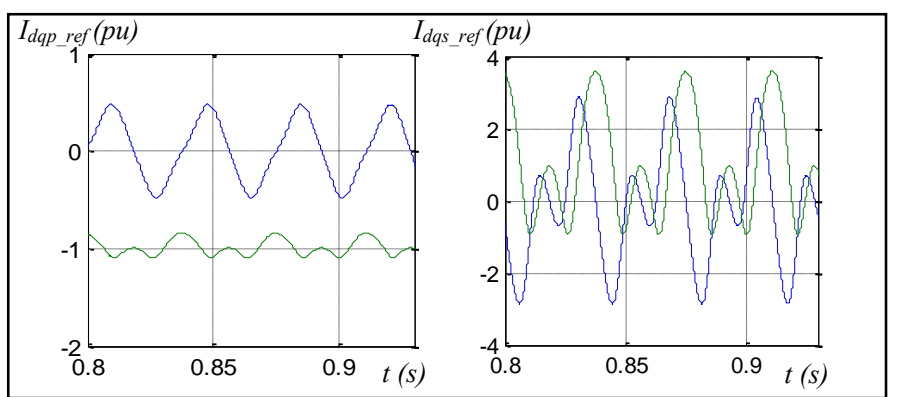

Figure 7(b). Référence de courant (en p.u.) avec une phase ouverte dans le référentiel de Park

\subsection{Contrôle non linéaire}

\subsubsection{Contrôle de courant par hystérésis à bande fixe.}

La commande par hystérésis à bande fixe (figure 8) consiste à maintenir le courant à l'intérieur d'une bande encadrant sa référence. La commande se fait alors sur chacune des phases par rapport à une référence calculée. L'écart existant entre le courant mesuré et le courant de référence est comparé à une bande fixe, appelée bande d'hystérésis $(H B)$. Chaque sortie de la bande entraîne un changement de la configuration des interrupteurs. Cette technique de régulation présente l'avantage d'être rapide, robuste et facile à mettre en œuvre. En revanche dans ce cas de contrôle du courant, la fréquence de commutation dépend essentiellement de la dérivée du courant de consigne. L'amplitude de la dérivée n'est donc pas maîtrisée et la fréquence de commutation n'est pas donc fixe. Ce point peut être particulièrement pénalisant dans le cas des systèmes de fortes puissances ou la fréquence de commutation est limitée à des valeurs de l'ordre du $\mathrm{kHz}$ du fait des caractéristiques des composants.

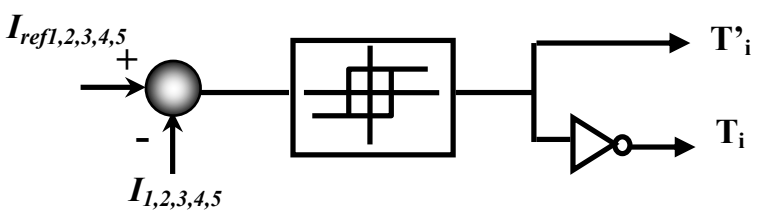

Figure 8. Schéma de la régulation du courant par hystérésis à bande fixe dans l a base naturelle 


\section{5 .2.2. Contrôle de courant par hystérésis à bande adaptative.}

Cette technique de modulation (figure 9) consiste également à réduire les écarts entre les courants de références et les courants absorbés par le moteur, et cela à fréquence constante. La commande hystérésis à bande adaptative est fondée sur un réglage dynamique de la bande d'hystérésis pour que la fréquence de commutation demeure constante (Rahman et al. 1997). Cette stratégie a été par exemple utilisée pour le contrôle des courants d'une machine triphasée dans (Seguier, 1998) et (Bose, 1990).

Pour une fréquence fixe désirée, l'algorithme de calcul réactualise la largeur de la bande d'hystérésis $H B_{a}$ en fonction des références de courant et des fem $e$. Par une méthode analogue à celle proposée dans les références pour une machine triphasée [Bose, 1990), l'expression de la bande adaptative est donnée par [10]. La figure 8 montre le schéma de contrôle du courant par hystérésis à bande adaptative.

$H B_{a}=\frac{a^{\prime} \cdot V_{d c}}{4 \cdot f_{c} \cdot L_{s}}\left[1-\frac{L_{s}^{2}}{a^{\prime 2} V_{d c}^{2}}\left(\frac{e}{L_{s}}+\frac{d I_{r e f}}{d t}\right)^{2}\right]$

$V_{d c}$ est ici la tension du bus continu, $f_{c}$ la fréquence de commutation, $L_{s}$ l'inductance d'une phase statorique et $a$ ' est coefficient moyen de la tension de sortie de l'onduleur issu de (Delarue et al. 2003). La méthodologie de calcul de cette bande adaptative est présentée plus en détail en annexe. Il est à noter que cette régulation par hystérésis à bande fixe ou adaptative est effectuée dans la base naturelle comme indiqué aux figures 8 et 9 .

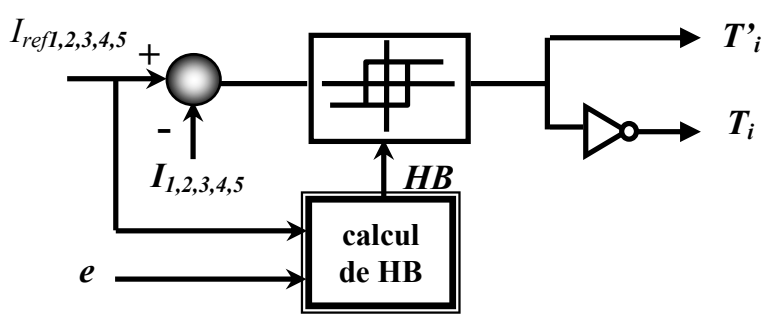

Figure 9. Schéma de la régulation du courant par hystérésis à bande adaptative dans la base naturelle

\section{Résultats de Simulation.}

Nous avons simulé en utilisant Matlab/ Simulink le comportement de la machine pentaphasée dont nous disposons avec la stratégie de commande étudiée pour le calcul des courants de références optimaux. Cette machine a les caractéristiques 
suivantes $\left(\Lambda_{\mathrm{p}}=1.5 \mathrm{mH}, \Lambda_{\mathrm{s}}=0.97 \mathrm{mH}\right)$. La répartition harmonique de la FEM est celle présentée au paragraphe II (tableau I). Cette machine est associée à un onduleur pentaphasé à IGBT piloté en MLI à $12 \mathrm{kHz}$. La bande du régulateur par hystérésis à bande auto adaptative est alors calculée pour cette fréquence de commutation, $\mathrm{f}_{\mathrm{c}}$, de $12 \mathrm{Khz}$ selon [10]. Dans le cas de contrôle par hystérésis à bande fixe, la bande fixe $2 H B(0,2$ p.u $)$ est égale à la valeur maximale de la bande adaptative $\left(2 H B_{a \max }\right)$.

\subsection{Mode normal}

Les figures 10, 11 et 12 montrent respectivement les résultats obtenus en utilisant un régulateur PID puis un régulateur par hystérésis à bande fixe et bande adaptative en mode sans défauts. On constate alors que nous avons un bon suivi de trajectoires de courants de références avec l'obtention d'un couple constant pour les 3 types de régulation. L'erreur $(\delta)$ de suivi en régime permanent est importante pour le contrôle par hystérésis à bande fixe et négligeable dans le cas à bande adaptative. Celle-ci dépend directement de la largeur de la bande fixe et se répercute sous forme d'ondulation excessive du courant de charge autour de sa référence. Ce qui se traduit par une augmentation significative des pertes supplémentaires.

L'objectif du contrôle étant de minimiser ces pertes pour un couple donné. A cet effet, le contrôle par PID et hystérésis à bande adaptative apparaissent alors beaucoup plus performants en mode normal. Elles permettent d'obtenir un couple de bonne qualité avec moins de 10\% d'ondulation (tableau 2).

\begin{tabular}{|l|l|l|}
\hline & $\mathrm{PJ}(\mathrm{pu})$ & $\Delta \mathrm{C}_{\max }(\mathrm{pu})$ \\
\hline PID & 1,02 & 0.1 \\
\hline Hystérésis & 1,02 & 0.1 \\
\hline $\begin{array}{l}\text { Hystérésis bande auto } \\
\text { adaptative }\end{array}$ & 1 & 0.05 \\
\hline
\end{tabular}

Tableau 2. Pertes Joule et ondulation de couple électromagnétique pour le mode sans défaut

\section{2. Mode de défaut}

\subsubsection{Maintien du couple nominal lors de l'apparition d'un défaut.}

Tout d'abord une première configuration est étudiée où lors de l'apparition d'un défaut on maintient constant le couple correspondant au mode normal.

Les figures 13, 14 et 15 représentent respectivement les résultats de simulation obtenus avec 2 phases consécutives ouvertes (phase 1 et 2). Ce mode de défaut 
correspond à la configuration la plus critique et est étudié pour des stratégies utilisant les différents contrôleurs à savoir : un régulateur PID et des régulateurs par hystérésis à bande fixe et à bande adaptative. Il est à noter que l'apparition de ces défauts se traduit par une augmentation significative des courants crêtes et donc des pertes Joule dans les phases si l'on souhaite maintenir le couple nominal.

La figure 16 illustre une vue rapprochée des courants de phases et de leurs références pour le contrôle par hystérésis (figure 16 (b) et (c)) et pour le régulateur PID (figure 16 (a)).

Dans ce cas de défaut, le courant de référence est plus important qu'en mode normal et a une dynamique rapide. La régulation PID, du fait de sa lenteur, trouve alors ces limites en termes de suivi en particulier lorsque les formes d'onde des courants de référence présentent des pentes raides. De ce fait, le courant réel est retardé par rapport à sa référence (figure 13 et 16(a)), l'erreur devient alors plus importante induisant des pulsations de couples et à moindre échelle des pertes supplémentaires. Ainsi, on obtient une ondulation de couple $\Delta \mathrm{C}_{\max }$ de $31 \%$ et des pertes Joule égales à 6,74 fois les pertes Joule obtenues en mode normal (dans le cas de la régulation PID).

L'utilisation d'un contrôle par hystérésis à bande fixe, permet de s'affranchir des problèmes de retard du courant réel par rapport sa référence inhérent au régulateur PID. Cependant le fait d'utiliser une largeur de bande qui conduit à une fréquence de commutation inférieure ou égale à la fréquence d'utilisation de l'onduleur de $12 \mathrm{kHz}$. L'usage de ce régulateur conduit à une ondulation du courant importante (figure14 et 16(b)). Il en résulte ainsi une ondulation de couple $\Delta \mathrm{C}_{\max }$ de $29 \%$ et des pertes Joule de 6,95 fois les pertes Joule obtenues en mode normal dans le cas du défaut de deux phases consécutives.

Le contrôle par hystérésis à bande adaptative quand à lui, (on maintient alors la valeur de bande fixe égale à la valeur maximale de la bande adaptative), permet à la fois d'avoir un très bon suivi de consigne avec une faible erreur et une minimisation des ondulations de couples (figures 15 et 16(c)). Les pertes Joule sont alors seulement de 6,6 fois les pertes en mode normal (dans le cas de la régulation PID) et l'ondulation de $10 \%$. On constate alors que l'ondulation de couple est divisée par 3 et que les pertes Joule sont diminuées de plus de 5\% par rapport au cas précédent.

Ces résultats en termes d'ondulation de couple et de pertes Joule sont synthétisés par le tableau 3.

Le tableau 4 quand à lui synthétise les résultats obtenus dans le cas du défaut d'une phase. La aussi le contrôle par hystérésis à bande auto adaptative apparaît comme particulièrement performant. Il conduit à une minimisation de l'ondulation de couple et dans une moindre mesure des pertes Joule. Il est à noter que, dans ce cas, la commande par PID présente des performances comparables à la commande par hystérésis à bande fixe car les dynamiques des références de courant sont moins élevées que dans les cas ou les deux phases sont en défaut. 


\begin{tabular}{|l|l|l|}
\hline & PJ $(\mathrm{pu})$ & $\Delta \mathrm{C}_{\max }(\mathrm{pu})$ \\
\hline PID & 6,74 & 0.31 \\
\hline Hystérésis & 6.95 & 0.29 \\
\hline $\begin{array}{l}\text { Hystérésis à bande auto } \\
\text { adaptative }\end{array}$ & 6.6 & 0.1 \\
\hline
\end{tabular}

Tableau 3. Pertes Joule et ondulation de couple électromagnétique pour le cas de deux phases consécutives en défaut.

\begin{tabular}{|l|l|l|}
\hline & $\mathrm{PJ}(\mathrm{pu})$ & $\Delta \mathrm{C}_{\max }(\mathrm{pu})$ \\
\hline PID & 1,36 & 0.12 \\
\hline Hystérésis & 1.38 & 0.12 \\
\hline $\begin{array}{l}\text { Hystérésis à bande auto } \\
\text { adaptative }\end{array}$ & 1.35 & 0.05 \\
\hline
\end{tabular}

Tableau 4. Pertes Joule et ondulation de couple électromagnétique pour le cas d'une phase en défaut.

\subsubsection{Maintien des pertes Joule nominales lors de l'apparition d'un défaut.}

Dans une deuxième configuration nous avons étudié la stratégie de commande qui consiste à maintenir les pertes Joule, associées aux nouveaux courants de référence, constantes et égales aux pertes en mode normal lors de l'apparition d'un défaut.

Dans cette configuration un nouveau couple de référence est recalculé pour limiter les pertes Joule à leur valeur nominale. Ainsi le nouveau couple de référence est alors obtenu en multipliant le couple de référence précédent par la racine carrée du ratio entre les pertes Joule en mode normal et les pertes Joule obtenues en défaut au paragraphe précédent.

Cette stratégie permet de maintenir la température de la machine à sa valeur nominale et d'éviter ainsi une dégradation liée au défaut avec un fonctionnement à couple réduit. Les tableaux 5 et 6 présentent la synthèse des résultats de simulation dans le cas du défaut d'une phase et de deux phases non adjacentes. Dans les deux cas le couple est alors réduit de manière significative. En effet le couple est alors réduit à $0,85 \mathrm{pu}$ pour le cas d'une phase ouverte et à $0,74 \mathrm{pu}$ avec deux phases ouvertes. Il est également à noter que l'amplitude des courants est quand à elle augmentée ce qui doit conduire logiquement à l'utilisation de composants surdimensionnés au niveau de l'onduleur surdimensionné pour les modes dégradés 
(Imax $=1,6 \mathrm{pu}$ pour une phase ouverte, et $\operatorname{Imax}=1,47 \mathrm{pu}$ pour deux phases ouvertes) Comme dans le cas précédent la stratégie de commande utilisant un contrôle par hystérésis à bande auto adaptative s'avère particulièrement performante car elle permet de minimiser les ondulations de couple en assurant un très bon suivi des références de courant en mode de défaut.

\begin{tabular}{|l|l|l|}
\hline & $\mathrm{PJ}(\mathrm{pu})$ & $\Delta \mathrm{C}_{\max }(\mathrm{pu})$ \\
\hline PID & 1.02 & 0.12 \\
\hline Hystérésis & 1.02 & 0.12 \\
\hline $\begin{array}{l}\text { Hystérésis à bande auto } \\
\text { adaptative }\end{array}$ & 1 & 0.08 \\
\hline
\end{tabular}

Tableau 5. Pertes Joule constantes : Mode Dégradé avec une phase ouverte, $C=0.85 \mathrm{pu}, \mathrm{I}=1.6 \mathrm{pu}$

\begin{tabular}{|l|l|l|}
\hline & $\mathrm{PJ}(\mathrm{pu})$ & $\Delta \mathrm{C}_{\max }(\mathrm{pu})$ \\
\hline PID & 1.02 & 0.12 \\
\hline Hystérésis & 1.02 & 0.12 \\
\hline $\begin{array}{l}\text { Hystérésis auto } \\
\text { adaptative }\end{array}$ & 1 & 0.08 \\
\hline
\end{tabular}

Tableau 6. Pertes Joule constantes : Mode Dégradé avec deux phases ouvertes (1et 3), $C=0.74 p u, \operatorname{Imax}=1,47 p u$ 


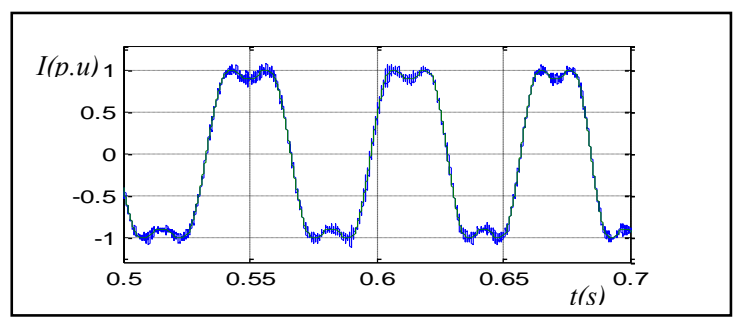

(a)

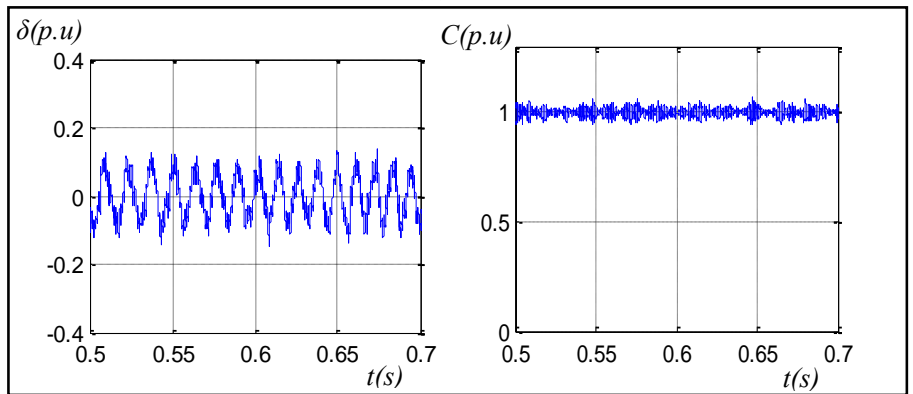

Figure 10.

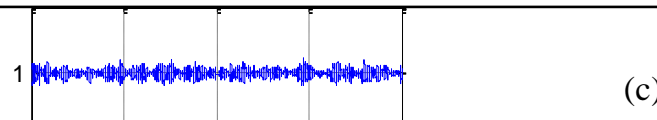

(b) erreur ${ }^{0} \bar{c}$

vvec régulateur PID : (a) courant statorique, urant dans la phase 2, (c) couple. 


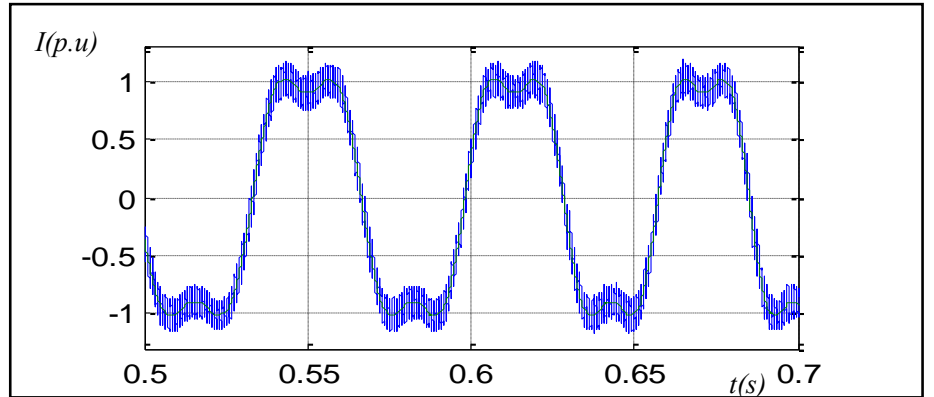

(a)
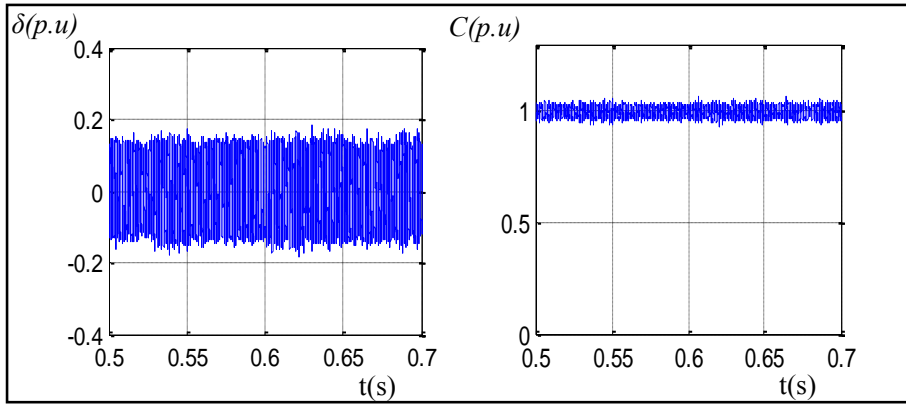

(b)

(c)

Figure 11. Configuration sans défaut avec régulation par hystérésis à bande fixe :(a) courant statorique, (b) erreur de suivi de la référence de courant dans la phase 2, (c) couple 


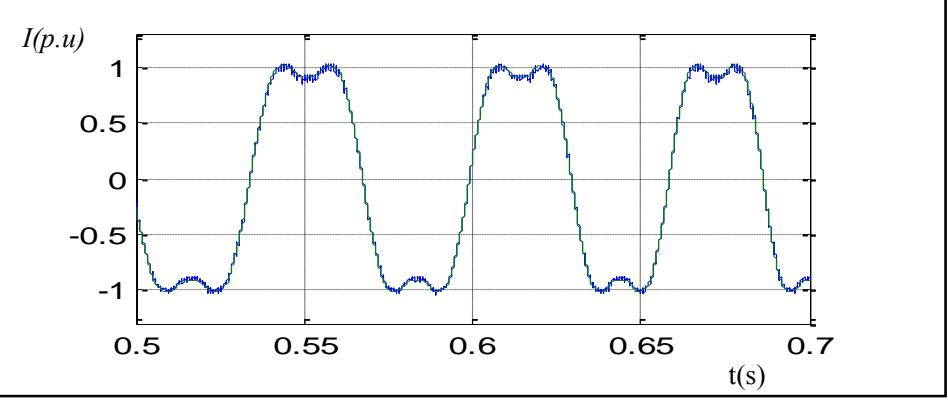

(a)

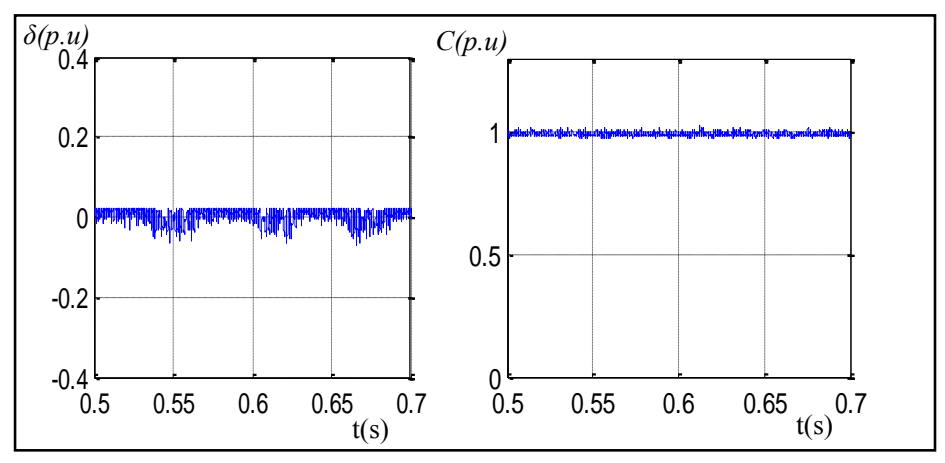

Figure 12. Configuration sans défaut avec régulation par hystérésis à bande adaptative : (a) courant statorique, (b) erreur de suivi de la référence de courant dans la phase 2, (c) couple. 


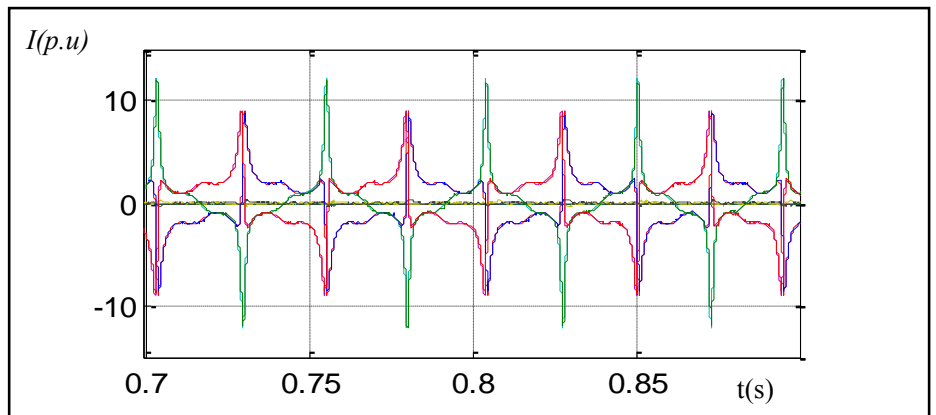

(a)

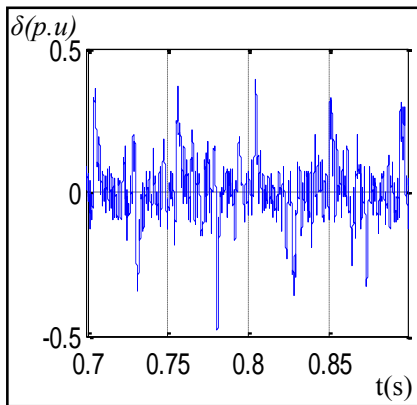

(b)

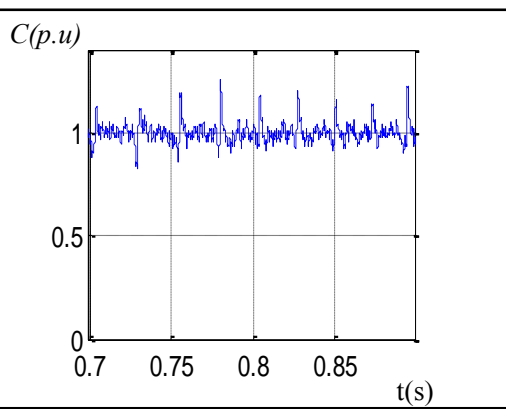

(c)

Figure 13. Configuration avec 2 phases consécutives ouvertes avec régulation PID:(a) courant dans les phases 1,2, 3 4,5, (b) erreur de suivi de la référence de courant dans la phase 2, (c) couple. 


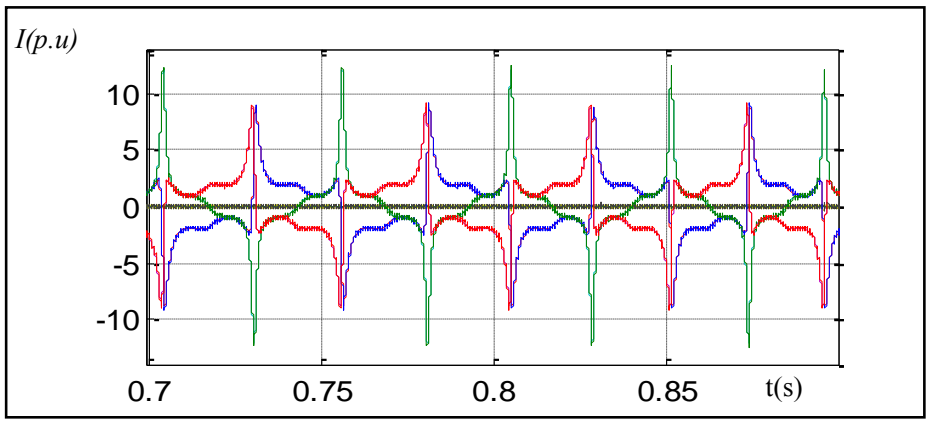

(a)
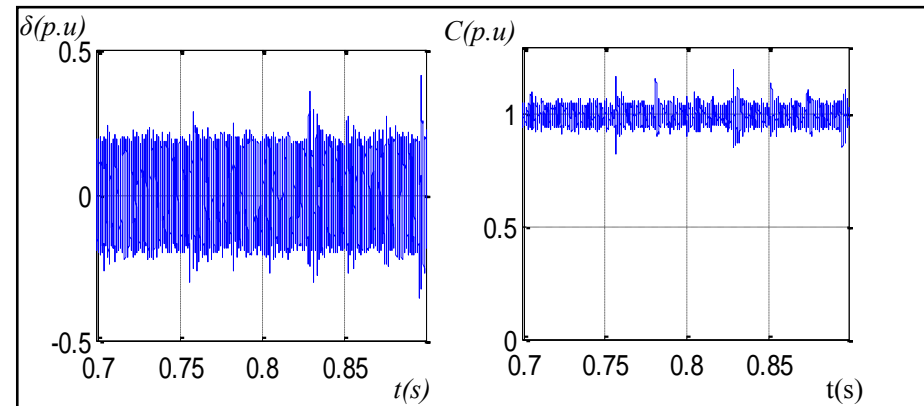

(b)

(c)

Figure 14. Configuration avec 2 phases consécutives ouvertes avec régulation par hystérésis à bande fixe:(a) courant dans les phases 1,2, 3 4,5, (b) erreur de suivi de la référence de courant dans la phase 2, (c) couple 


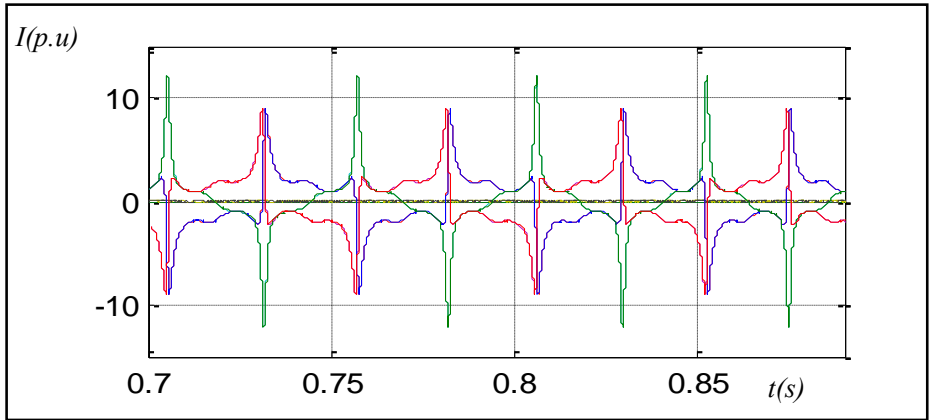

(a)

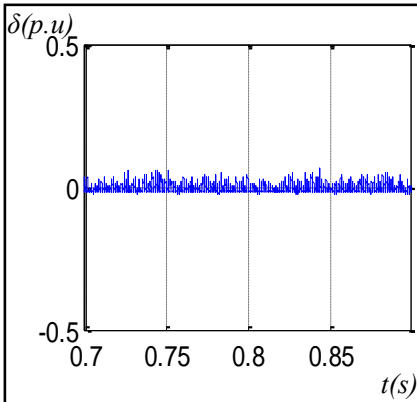

(b)

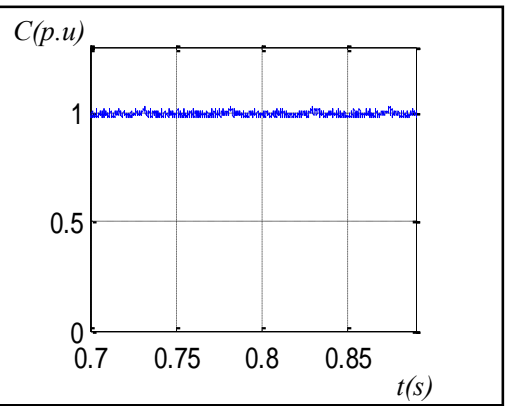

(c)

Figure 15. Configuration avec 2 phases consécutives ouvertes commande par (hystérésis à bande adaptative) : (a) courant dans les phases 1,2, 3 4,5, (b) erreur de suivi de la référence de courant dans la phase 2 , (c) couple 


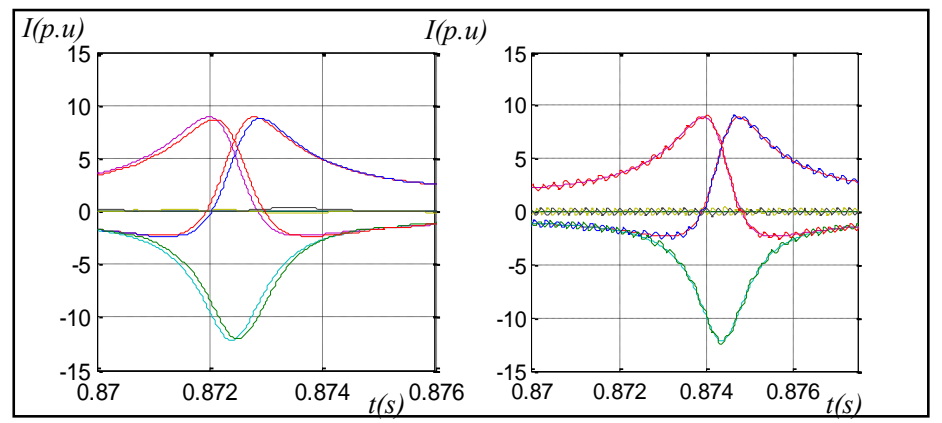

(a)

(b)

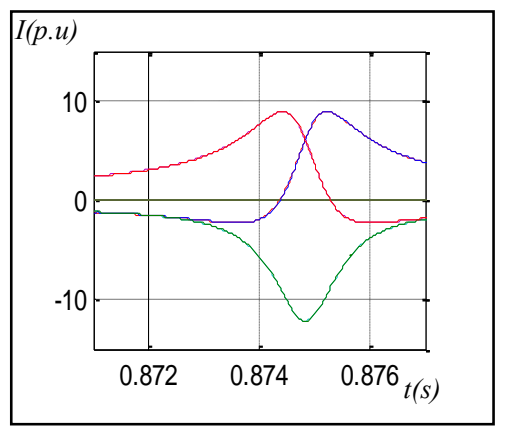

(c)

Figure 16. Vue rapprochée des courants de phases et de leurs références en (pu) pour : (a) $P I D$, (b) hystérésis à bande fixe, (c) hystérésis à bande adaptative.

\section{Conclusion}

Dans ce travail nous proposons une stratégie de génération des références de courants d'une machine polyphasée à aimants permanents associée à un onduleur MLI. Cette stratégie permet à la fois de traiter les modes dégradés (de type ouverture de phase) et normaux en utilisant le même algorithme de génération des courants de références en ligne, et la même architecture de commande.

L'objectif de cette stratégie de commande est alors de minimiser les pertes Joule à couple donné, en supprimant les ondulations du couple dues à la déconnexion d'une ou plusieurs phases d'alimentation d'une machine polyphasée.

En utilisant cette stratégie de génération des courants de références, un mode de régulation utilisant des transformations de Park dédiées à chacune des machines 
fictives déterminées selon la théorie multi machines et des régulateurs de type PID est comparée à une stratégie de régulation utilisant un contrôle direct des courants par hystérésis à bande fixe et à bande adaptative.

Dans le cas du mode normal la première stratégie s'avère particulièrement performante. Cependant, il est à noter que l'emploi de correcteurs de type PID associé à des transformations de Park est discutable dans les modes de défauts. En effet, les grandeurs selon les axes d et q dans les machines fictives ne sont alors pas constantes. L'augmentation de la vitesse du système se traduit alors par des difficultés de suivi des références de courant et donc par un couple moins maîtrisé et des pertes Joule supplémentaires. Dans ce cas, le contrôle par hystérésis à bande fixe peut paraître plus adapté car il permet d'avoir un meilleur suivi des références mais la fréquence de commutation de l'onduleur est alors variable ce qui peut être pénalisant pour des applications de fortes puissance ou peut se traduire par des pulsations de couple si l'on contraint la bande de manière à limiter la fréquence de commutation de l'onduleur à des valeurs acceptables. Afin d'améliorer les performances du système en particulier pour les régimes de haute vitesse, un contrôle par hystérésis à bande adaptative dont la fréquence de commutation est quasi constante a été proposé et comparé avec un contrôle par PID et par hystérésis à bande fixe. La commande proposée conjugue les avantages des commandes par hystérésis à bande fixe et par PID. Elle permet de manière simple d'obtenir un bon suivi des références et de bonnes performances en termes d'ondulation de couple et de pertes Joule tout en permettant de maitriser la fréquence de commutation. Elle permet donc de très bonnes performances dans les modes normaux et de défaut et ne nécessite pas de reconfiguration du système, mais le contrôle de la bande est sensible aux paramètres de la machine.

\section{References}

Parsa L, Toliyat H., «Five- Phase Permanent - Magnet Motor Drives » IEEE Transactions on Industry applications », vol. 41, n¹, 2005, P 30 - 37, ISSN: 0093-9994.

Dwari S, Parsa L., « An Optimal Control Technique for Multiphase PM Machines Under Open Circuit Fautls », IEEE Transactions on Industry Electronics, vol. 55.n`5, May 2008, p 1988 - 1995,

Levi E, Martin J, Slobodan N.V, Toliyat H., « Operating Principles of a Novel Multiphase Multimotor Vector - controlled Drive », IEEE Transactions on Energy Conversion, vol. 19, $\mathrm{n}^{\circ} 3$, September 2004, p 508-517.

Locment F, Semail E, Kestelyn X., « Vectorial Approach Based Control of a seven- phase Axial Flux Machine Designed for Fault Operation », IEEE 2008.

Bolognani S, Zordan M, Zigliotto M., «Experimental fault-tolerant control of a PMSM drive », IEEE Transactions on Industrial Electronics, vol. 47, n5, Oct 2000 , p. 1134 - 1141 ISSN: 0278-0046 
Martin J-P, Meibody-Tabar F, Davat B., « Multiple-phase permanent magnet synchronous machine supplied by VSIs working under fault conditions », IEEE-IAS annual meeting, Roma, october 2000, CD-ROMX.

Martin J-P, Pierfederici S, Meibody-tabar F, Letellier R., « Synthèse des méthodes de filtrage du couple des MSAP polyphasées en modes normal et dégradé », Revue Internationale de Génie Electrique, vol. 10, n 1-2, Avril 2007, p. 117-149.

Kestelyn X., Modélisation vectorielle multimachines pour la commande des ensembles convertisseurs machines polyphasés, Thèse de doctorat, Université de Lille1, 2003.

Parsa L, Toliyat H., « Sensorless Direct Torque Control of Five Phase Interior Permanent Magnet Motor Drives », IEEE Transactions on Industry applications, vol. 43, $\mathrm{n}^{\circ} 4$, July/August 2007, p 952 - 959.

Semail E, Kestelyn X, Bousscayrol A., « Sensitivity of a 5 phase Brushless DC machine to 7th harmonic of back-electromotrice force », PESC 2004 IEEE Power Electronics, Germany, CD- ROM.

Kestelyn X., «Generation of on line optimal current references for multi-phase permanent magnet machines with open circuit phases », Proceedings IEMDC, 2009, p 689-694.

Kestelyn, X.; Semail, E.; , «A Vectorial Approach for Generation of Optimal Current References for Multiphase Permanent-Magnet Synchronous Machines in Real Time» Industrial Electronics, IEEE Transactions on, vol.58, no.11, pp.5057-5065, Nov. 2011

Semail. E; F. Meibody-Tabar. F; Benkhoris. M. F; Razik; H. Pietrzak-David, M. Monmasson; Bouscayrol. A; Davat. B; Delarue. Ph; de Fornel. B, Hautier. J. P ; Louis. J. P, Pierfederici. S, «Représentations SMM de machines polyphasées», RIGE, Revue Internationale de Génie Electrique, vol. 8 n²/2005, pp. 221-239 ; ISSN : 1295-490X

Seguier G., Les convertisseurs de l'électronique de puissance : la conversion alternative continue, ISBN2-85206-X-1998, Dunod

Bose B.K., « An adaptive hysteresis- band current control technique of a voltage fed PWM inverter for machine drive system », IEEE Trans. on Ind. Electronics, vol. 37, n5, 1990, p. $402-408$.

Rahman K.M, Khan M.R, Choudhury M.A., « Variable band hysteresis current controllers for PWM voltage source inverters », IEEE Trans on Power Electronics, vol. 12, n 6, 1997, 964-970.

Delarue P, Bouscayrol A, Semail E., « Generic control method of multi-leg voltage-sourceconverters for fast practical implementation », IEEE Transaction on Power Electronics, vol. $18, \mathrm{n}^{\circ} 2,2003$,p. 517-526. 
Annexe : Méthode de calcul de la bande pour un contrôle par hystérésis à bande adaptative.

Pour déterminer l'expression de la bande, on se base sur une machine synchrone à aimant permanent triphasée commandée par un onduleur triphasé (Seguier, 1998)(Bose, 1990). Le schéma de la figure A1 illustre l'incrémentation du courant ' $i$ ' autour de la grandeur de référence ' $i$ ' ' pendant une période $T_{c}$ de modulation (par analogie avec la modulation utilisant la comparaison d'une onde de modulation avec une onde de référence). On peut considérer la période de modulation ' $t_{1}+t_{2}$ ' , on suppose que la FEM est positive est constante, et les interrupteurs $T_{1}$ et $T_{1}$ ' se ferment respectivement pendant des durées $\mathrm{t}_{1}$ et $\mathrm{t}_{2}$, les valeurs de la tension $v_{s}$ sur la première phase sont égales à $0,1 / 3$ ou $2 / 3$ de $V_{d c}$ lorsque $\mathrm{T}_{1}$ est fermé et à $0,-1 / 3$ ou $2 / 3$ de $V_{d c}$ quand $\mathrm{T}_{1}$ 'est fermé (Bose, 1990). L'expression générale de l'incrémentation du courant $\Delta \mathrm{HB}$ durant la phase ascendante $\left(\mathrm{T}_{1}\right.$ passant) est donnée par:

$$
\Delta H B=\frac{t_{1 n}}{L_{s}}\left(a V_{d c}-e^{\prime}\right)-t_{1 n} \cdot \frac{d i^{*}}{d t}
$$

D'où la sommation du courant total est :

$$
\begin{aligned}
2 H B= & \sum_{n} \Delta H B=\sum_{n}\left[t_{n 1}\left(\frac{\left(a V_{d c}-e^{\prime}\right.}{L_{s}}\right)-t_{1 n} \cdot \frac{d i^{*}}{d t}\right] \\
& =-t_{1} \cdot\left(\frac{e^{\prime}}{L_{s}}+\frac{d i^{*}}{d t}\right)+\frac{1}{L_{s}} \sum_{n}\left(t_{1 n} \cdot a \cdot V_{d c}\right)
\end{aligned}
$$

Pendant la phase descendante ( $\mathrm{T}_{1}$ 'passant) l'incrémentation du courant se met sous la forme.

$$
-\Delta H B=\frac{t_{2 n}}{L_{s}}\left(a V_{d c}+e^{\prime}\right)-t_{2 n} \cdot \frac{d i^{*}}{d t}
$$

Cependant, HB est exprimé par :

$$
-2 H B=-t_{2} \cdot\left(\frac{e^{\prime}}{L_{s}}+\frac{d i^{*}}{d t}\right)-\frac{1}{L_{s}} \sum_{n}\left(t_{2 n} \cdot a \cdot V_{d c}\right)
$$




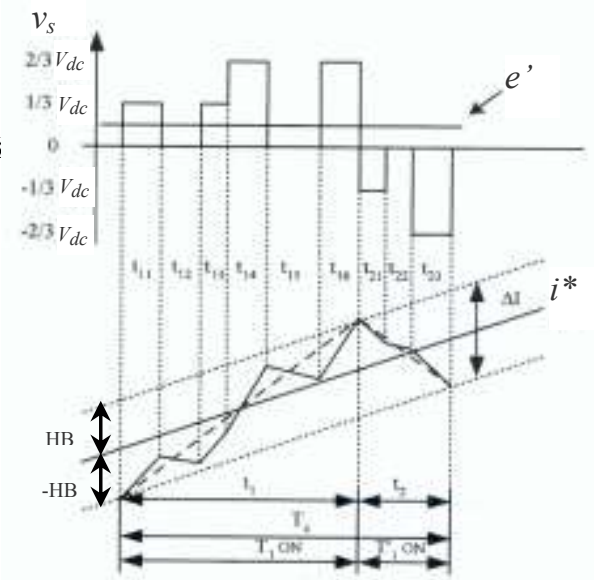

Figure A1. Représentation de la tension en sortie de l'onduleur et le courant dans cycle de modulation tirée de (Bose, 1990)

Le courant moyen pendant chacune des phases est représenté en pointillé sur la figure 7. Les pentes correspondantes sont déterminées en considérant la valeur moyenne de la tension délivrée par l'onduleur $V_{s}$ sur une période d'échantillonnage.

Le second terme de [A2] et [A4] peut s'écrire :

$$
\left.\begin{array}{l}
\sum_{n} t_{1 n} \cdot a \cdot V_{d c}=t_{1} \cdot a^{\prime} \cdot V_{d c} \operatorname{avec} a^{\prime}=\sum_{n}\left(t_{1 n} \cdot a\right) / t_{1} \\
\sum_{n} t_{2 n} \cdot a \cdot V_{d c}=t_{2} \cdot a^{\prime \prime} \cdot V_{d c} \text { avec } a^{\prime \prime}=\sum_{n}\left(t_{2 n} \cdot a\right) / t_{2}
\end{array}\right\}
$$

Les coefficients a' et a', représentent la valeur moyenne du paramètre ' $a$ ' sur les deux intervalles de temps. Pour simplifier, nous prenons a' et a" égaux. Dans ce cas, la combinaison de [15], [17] et [18] conduit à l'expression simplifiée de HB :

$$
H B=\frac{a^{\prime} \cdot V_{d c}}{4 \cdot f_{c} \cdot L_{s}}\left[1-\frac{L_{s}^{2}}{a^{\prime 2} V_{d c}^{2}}\left(\frac{e^{1}}{L_{s}}+\frac{\mathrm{di}_{\mathrm{s}}^{*}}{d t}\right)^{2}\right]
$$

Pour une fréquence fixe désirée, l'algorithme de calcul réactualise la largeur de la bande d'hystérésis HB donnée par [A6]. Cette équation est valable dans le cas de la machine n-phases. La valeur de $a$ ' dépend de la topologie d'alimentation (Rahman et al. 1997]. Dans notre cas, les phases de la machine sont couplées en étoile sans neutre (figure 1), donc on peut prendre $a^{\prime}=1 / 5$ (Philippe et al. 2003). La figure 9 montre le schéma de contrôle du courant par hystérésis à bande adaptative. 